\title{
Thin Filament Changes during In Vivo Rat Heart Development
}

\author{
THOMAS J. L'ECUYER, DOUGLAS SCHULTE, AND JIM JUNG-CHING LIN
}

Department of Pediatrics [T.J.L.] and Department of Biology, [D.S., J.J.-C.L.], University of Iowa, Iowa City, Iowa 52242

\begin{abstract}
Developmental differences in myocardial performance are known to exist. It is likely that the profile of protein isoforms present on the developing thin filament contributes to these observed differences. We have prepared thin filaments from developing and mature rat hearts by using an immunoprecipitation procedure developed in our laboratory. Analysis of these isolated thin filaments by Western immunoblots and two-dimensional gel electrophoresis demonstrates troponin $I$ and troponin $T$ isoform switching on the developing thin filament. Troponin $I$ isoform switching begins by embryonic $d 18$ and is complete before the 3rd postnatal wk. Troponin $\mathrm{T}$ isoform switching begins between embryonic $\mathrm{d} 18$ and birth and is complete between the 2nd and 3rd postnatal wk. The degree of phosphorylation of tropomyosin in thin filaments appears to be developmentally regulated, decreasing with advancing age. Nonmuscle isoforms of tropomyosin are also detectable in thin filaments from developing and mature rat hearts. These phenomena (troponin isoform switching, the degree of phosphorylation of tropomyosin, and the presence of nonmuscle isoforms of tropomyosin on cardiac thin filaments) likely play a role in the function of immature thin filaments and in the assembly of mature thin filaments. (Pediatr Res 30: 232-238, 1991)
\end{abstract}

\section{Abbreviations}

Tm, tropomyosin

pTm, phosphorylated tropomyosin

MHC, myosin heavy chain

CL2-MF, tropomyosin-enriched microfilaments

Developmental differences in myocardial function have been observed in a variety of species, including dogs (1), rabbits (2), and rats (3). Myocardial function improves significantly in these species as development progresses toward adulthood. In addition, the inotropic response to isoproterenol (1), phosphodiesterase inhibitors (4), and calmidazolium (5), and the immediate decline in myocardial performance with acidosis (6) are more significant in adult than neonatal myocardium.

The functional properties of the contractile apparatus are likely derived from the composition of its constituent structural and regulatory proteins. Because cardiac muscle development involves the sequential and/or overlapping expression of protein isoforms, the developmental switching of isoforms may be a mechanism for observed functional changes $(7,8)$. Develop-

Received December 20, 1990; accepted May 10, 1991.

Correspondence: Thomas J. L'Ecuyer, M.D., Department of Pediatrics, University of Iowa, Iowa City, IA 52242.

Supported by NIH Grants GM40580 and congenital heart disease SCOR HL42266, a grant from the Muscular Dystrophy Association, and NIH Training Grant HL07413. mental isoform switching has been described for $\operatorname{MHC}(9,10)$, troponin $\mathrm{I}(11,12)$, and troponin $\mathrm{T}(13,14)$. The isoform switch of MHC (from $\alpha$-MHC to $\beta$-MHC) may be functionally important, in that the calcium-activated myosin ATPase activity of $\alpha$ $\mathrm{MHC}$ is higher than $\beta$-MHC, resulting in a more rapid velocity of shortening (15). Still, the absence of an MHC isoform switch in many mammals (including humans) makes it unlikely that MHC isoform switching alone accounts for the observed developmental difference in myocardial function. Thus, our attention has turned to differences in the thin filament during heart development. Functional differences, although subtle, have been demonstrated between troponin $T$ isoforms (16), with the larger isoform having slightly greater calcium sensitivity in an in vitro reconstituted system. Functional differences between troponin I isoforms exist, with the adult isoform being more rapidly phosphorylated than the embryonic, and thereby possibly allowing more rapid release of calcium from troponin $\mathrm{C}$, with consequent relaxation $(17,18)$. Muscle Tm isoform switching is not seen in all species during heart development, and troponin $\mathrm{C}$ appears to exist as a single protein throughout development.

The role of developmental protein isoforms is not well understood. Possibilities include a role in assembly of developing microfilaments, in the contraction of embryonic thin filaments, or both. In an attempt to learn more about the significance of isoform switching on the thin filament, we have immunoprecipitated thin filaments from developing rat hearts with an antibody to skeletal muscle Tm (19). This antibody has been shown previously (19) to be able to isolate CL2-MF from embryonic chick skeletal $(19,20)$ and cardiac muscles $(20)$. These thin filaments were then analyzed by two-dimensional gel electrophoresis and Western immunoblots with antibodies we have developed to troponin $\mathrm{T}$ and troponin I. Western immunoblots of immunoprecipitated thin filaments were compared with those of intact heart tissue probed with the same antibodies. We have found that troponin $\mathrm{T}$ and troponin I isoform switching occurs on the developing thin filament. In addition, it appears that the degree of phosphorylation of Tm on the thin filament is developmentally regulated, decreasing as development progresses. Finally, we have detected nonmuscle isoforms of $\mathrm{Tm}$ on the developing (and mature) thin filament. This is new information that cannot be concluded from analysis of whole tissue. These results suggest that troponin $\mathrm{T}$ and troponin $\mathrm{I}$ isoform switching may contribute to developmental differences in myocardial function. The degree of phosphorylation of cardiac Tm may play a role in the assembly and/or function of developing thin filaments, as may the presence of nonmuscle isoforms of $\mathrm{Tm}$ on developing thin filaments.

\section{MATERIALS AND METHODS}

Purification of troponin I. Troponin I was purified from bovine left ventricle by the method of Potter (21). Briefly, the procedure involves preparation of an ether powder, a $1 \mathrm{M} \mathrm{KCl}$ extraction of the powder, and initial fractionation of troponin with am- 
monium sulfate. The initial extraction removes the majority of soluble proteases and myosin. The ammonium sulfate fractionation separates crude troponin from a fraction containing a high concentration of $\mathrm{Tm}$. The crude troponin was further purified with chromatography on CM Sephadex C-50-120 and DEAESephadex A-50 in the presence of $6 \mathrm{M}$ urea and $1 \mathrm{mM}$ EDTA to prepare troponin I. This troponin I was further purified by electrophoresis in a polyacrylamide gel followed by excision of the desired protein band and electroelution of the protein from gel slices (22).

Preparation of specific antibodies. A New Zealand White rabbit was immunized s.c. with bovine cardiac troponin I $(100 \mu \mathrm{g})$ emulsified with an equal volume of complete Freund's adjuvant. After $2 \mathrm{wk}$, the rabbit was boosted with $80 \mu \mathrm{g}$ of the same antigen emulsified in incomplete Freund's adjuvant. The animals were boosted three more times at 2- to 3- wk intervals and tested for antibody production. The antiserum thus obtained was characterized by Western immunoblot, by using a modification of the procedure of Towbin et al. (23), and by immunofluorescent staining of cryosections of rat ventricle and cultured rat heart myocytes, by the procedure of Lazarides and Weber (24). A polyclonal antibody to skeletal troponin I (GPI) was prepared as described above by injection of purified rabbit skeletal troponin I into a guinea pig. Mouse MAb CT3 against cardiac troponin T and CL2 against striated muscle Tm were prepared and characterized as described previously $(25,26)$.

Tissue preparation. To obtain myocardial tissue for Western immunoblot experiments, timed pregnancies were interrupted by killing pregnant females and hearts from fetal rats were harvested on ice. Animals were cared for by the animal care unit at the University of Iowa, and animals were anesthetized before being killed. The hearts from an entire litter of fetal or neonatal rats or single hearts of larger rats were homogenized with a Brinkman polytron for $45 \mathrm{~s}$ at speed 4 in $10 \mathrm{vol}$ of a cold buffer consisting of $10 \mathrm{mM}$ Tris (pH 8.0) and $1 \mathrm{mM}$ EGTA. The homogenate was centrifuged for $10 \mathrm{~min}$ at $5000 \mathrm{rpm}$; the pellet was suspended in sample buffer (100 mM DTT, 2\% SDS, 80 $\mathrm{mM}$ Tris [pH 6.8], $15 \%$ glycerol, and $0.006 \%$ bromphenol blue), heated for $3 \mathrm{~min}$ at $100^{\circ} \mathrm{C}$, and centrifuged in an Eppendorf centrifuge for $15 \mathrm{~min}$ at $4^{\circ} \mathrm{C}$, and the supernatant was collected.

Cardiac myocyte culture. Wistar rat hearts ( 1 to $3 \mathrm{~d}$ old) were used to establish cardiac myocyte cultures by the method of Borg et al. (27). Cultures were grown in $35-\mathrm{mm}$ dishes containing glass coverslips. The cardiac myocytes grown on these coverslips were used for immunofluorescent staining by the method of Lazarides and Weber (24).

Cryosections. To prepare cryosections of adult rat ventricles, a single adult rat was euthanized with ether and the heart was rapidly removed. The left ventricle was quickly frozen with liquid nitrogen after mounting in OCT compound. Sections $(6 \mu \mathrm{m})$ were cut on a microtome, fixed in cold acetone, and used for immunofluorescent staining.

One- and two-dimensional gel electrophoresis. A 12.5\% SDSPAGE was carried out as described by Laemmli (28) with a low concentration of bisacrylamide (29). Two-dimensional gel electrophoresis was performed by a modification (30) of O'Farrell's procedure (31).

Isolation of Tm-enriched microfilaments. We have previously shown that monoclonal antibody CL2 was able to specifically immunoprecipitate the skeletal CL2-MF from myotubes but not from fibroblasts $(19,26)$. Native myofilaments consist of thin and thick filaments; our isolation procedure produces thin filaments containing primarily actin, Tm, and the troponins. We have also shown that CL2 was able to isolate striated muscle CL2-MF from embryonic chick muscles (20). Therefore, CL2 antibody was used to isolate CL2-MF from embryonic and postnatal developing rat hearts by the procedure previously described (20). The resulting pellets (i.e. rat cardiac (CL2-MF) were dissolved in gel sample buffer for analysis by one- and twodimensional gels and by Western immunoblots.

\section{RESULTS}

Characterization of rabbit antisera to troponin I. Western immunoblot and immunofluorescence were used to characterize the rabbit antisera to troponin I (RTI). Figure 1 shows the results obtained from Western immunoblot of crude bovine troponin (lane 1) and adult rat heart homogenate (lane 2). A single band is recognized, with an apparent molecular mass of $30 \mathrm{kD}$. Immunofluorescent staining of cultured neonatal rat heart myocytes demonstrates a doublet on the I bands of the myofibrils (arrow in Fig. 2), which is a typical localization for troponin I on the myofibril. Immunofluorescent staining of frozen sections of adult rat ventricle also reveals I band localization (arrow in Fig. 3).

Troponin $T$ and troponin I isoform switching during development of rat heart. By using the RTI rabbit antiserum and CT3, we examined isoform switching of troponin I and troponin $T$ during rat heart development in whole heart homogenates. Embryonic rat heart contains a specific protein band with an apparent molecular mass just under $45 \mathrm{kD}$ that is recognized by CT3, representing the embryonic isoform of rat cardiac troponin $T$ (Fig. 4, lane 1). The smaller adult isoform appears within the first $24 \mathrm{~h}$ after birth (Fig. 4, lane 2), increases in proportion to the embryonic isoform as development progresses, and is the exclusive isoform present after approximately $3 \mathrm{wk}$ of postnatal development (Fig. 4, lane 6). The adult isoform of troponin I is first recognized in hearts at approximately $16 \mathrm{~d}$ of gestation (Fig. 4 , lane I). Our antibody to cardiac troponin I inconsistently recognizes a protein with an apparent molecular mass of $25 \mathrm{kD}$, the expected size of the embryonic isoform of troponin I (data not shown). As development progresses, there is an increase in the amount of adult cardiac troponin I detected by this antibody (compare, e.g. lane 1 with lane 7 in Fig. 4).

Troponin isoform switching during rat heart thin filament development. The location of troponin $\mathrm{T}$ and troponin I isoform switching within cardiac muscle, although assumed to take place on the developing thin filament, is not known. It is possible that knowing the location of the isoform switch may yield information about the potential roles of embryonic isoforms of these
A

A mido

Black

$\stackrel{0}{\infty} 12$

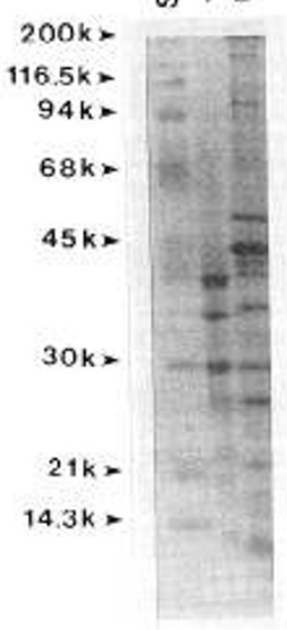

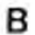

R T I
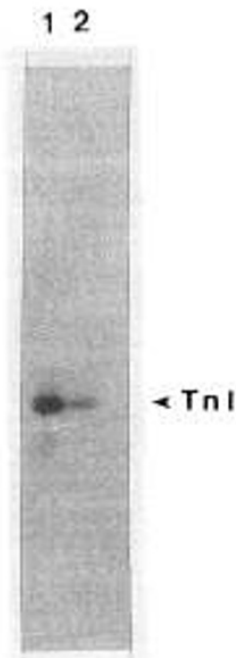

Fig. 1. Western immunoblot demonstrating specificity of anticardiac troponin I polyclonal antibody. After electrophoresis and transfer of proteins to nitrocellulose paper, the papers were either stained with amido black $(A)$ or reacted with the polyclonal antibody $\mathrm{RTI}(B)$ followed by ${ }^{125}$ I-labeled goat anti-rabbit IgG. Bound antibody was detected by autoradiography. Antibody binding is demonstrated to a specific protein $(T n I)$ in bovine crude troponin (lane 1 ) and adult rat heart homogenate (lane 2). Std, molecular weight standards. 

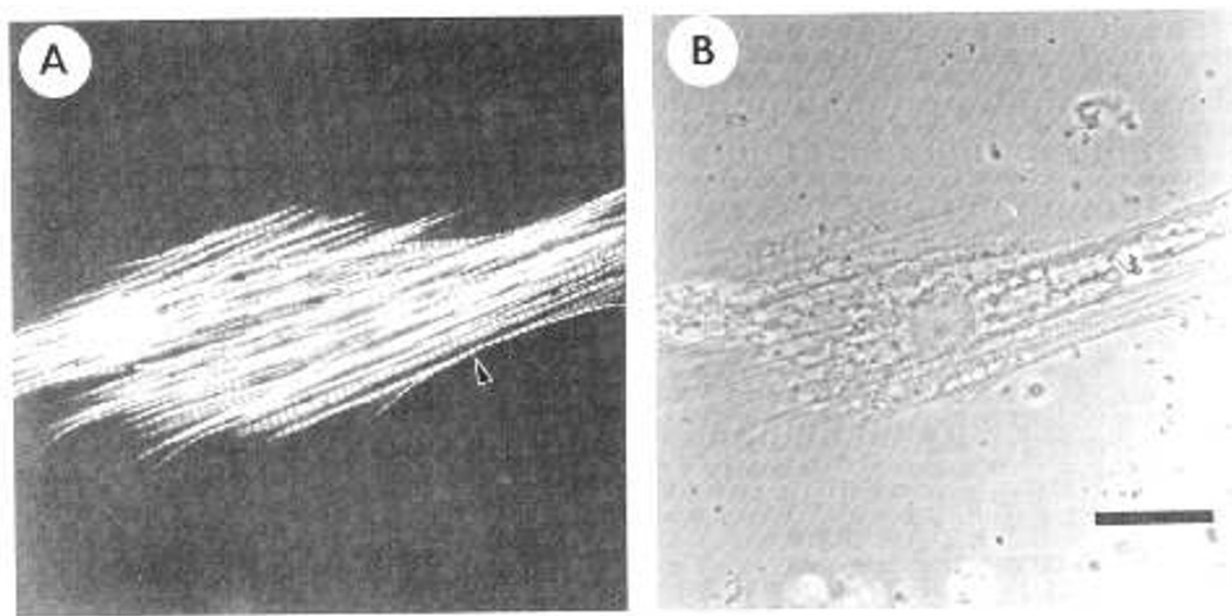

Fig. 2. Immunofluorescent staining of cultured rat heart myocytes with polyclonal antibody RTI. After fixation and permeabilization, cells were incubated with RTI followed by fluorescein-conjugated goat anti-rabbit IgG. $A$, Fluorescent micrograph. $B$, Phase-contrast micrograph. Note specific staining of I bands (arrow indicates single I band). Bar $=2 \mu \mathrm{m}$.
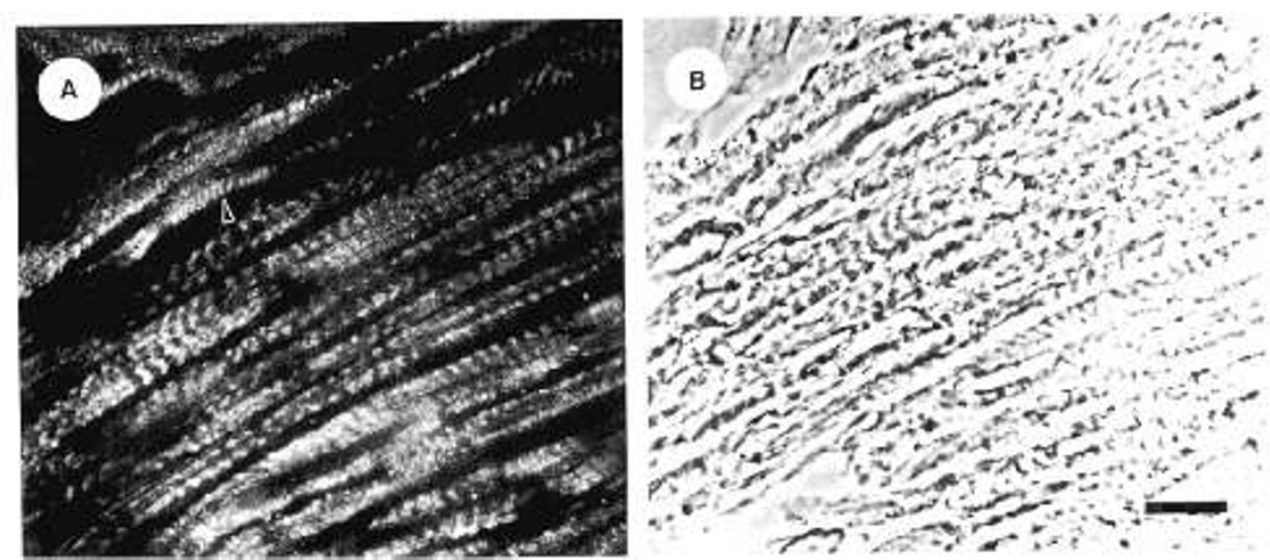

Fig. 3. Immunofluorescent staining of cryosection of adult rat ventricle with polyclonal antibody RTI. Sections $(6 \mu \mathrm{m})$ of tissue were cut from ventricle mounted in OCT compound, fixed in cold acetone, and incubated in RTI followed by fluorescein-conjugated goat anti-rabbit IgG. $A$, Fluorescent micrograph. B, Phase-contrast micrograph. Note specific staining of I bands (arrow indicates single I band). Bar $=1 \mu \mathrm{m}$.

proteins during heart development. Possible roles include assembly and contraction of developing microfilaments. To examine these possibilities, we immunoprecipitated thin filaments from developing and mature rat hearts by using an antibody (CL2) that reacts specifically with striated muscle Tm (19). This antibody has been shown to be able to selectively isolate striated muscle CL2-MF from developing chick embryos (20). The isolation procedure causes myosin-containing thick filaments to dissociate into myosin monomers or soluble oligomers by the addition of ATP, which is subsequently hydrolyzed. We have previously shown that our isolation procedure does not interfere with the binding of other actin binding proteins (including myosin) to the microfilament preparation (20). During incubation with antibody, some myosin monomers may bind to the microfilaments, explaining why isolated microfilaments contain myosin molecules. We call the product of this procedure CL2-MF because an antibody to $\mathrm{Tm}$ is used to precipitate this protein and other proteins associated with it (primarily thin filament proteins), not because $\mathrm{Tm}$ is isolated in greater quantity than other thin filament proteins. Thin filaments prepared in this fashion were examined by Western immunoblot using RTI, GPI, and CT3 antibodies. When these CL2-MF were examined with CT3, the larger embryonic isoform of troponin $\mathrm{T}$ is seen exclusively in the 20-d-old embryonic heart (Fig. 5B, lane 1). Within $24 \mathrm{~h}$ after birth, the smaller adult isoform appears (Fig. $5 \mathrm{~B}$, lane 2 ). As development progresses, the adult isoform increases in proportion to the embryonic isoform; the isoform switch is complete between the 2 nd and 3 rd postnatal wk (Fig. 5B, lane 6). Troponin
$\mathrm{T}$ isoform switching thus occurs on the developing thin filament. In Figure $5 C$, the same CL2-MF were examined with RTI antisera. The adult isoform of cardiac troponin $\mathrm{I}$ is first recognized by this antibody shortly after birth (Fig. $5 C$, lane 2 ), although this protein is recognized in homogenates from late embryonic hearts (Fig. 4, lane 1). Because it has been suggested (32) that the embryonic isoform of troponin I may be the same protein as slow skeletal troponin I, an antibody (GPI) to skeletal troponin I was used to examine the CL2-MF for the presence of this protein. A protein of apparent molecular mass of $25 \mathrm{kD}$ is recognized by GPI in microfilaments from 20 -d embryonic (Fig. 6, lane 1) and 7-d postnatal (Fig. 6, lane 2), but not 3-wk postnatal (Fig. 6, lane 3) hearts. The adult isoform of troponin I is also recognized in cardiac microfilaments from late gestation through adulthood ( $a$ in Fig. 6, lanes 1-3). A third band, intermediate in apparent molecular mass between the adult and embryonic isoforms of troponin I, is also detected at all ages examined. The identity of this protein is yet to be determined but may represent a troponin I proteolytic fragment or another troponin I isoform. Because we added DTT to our samples before electrophoresis of proteins, it is unlikely that this band represents incomplete reduction of sulfhydryl groups in troponin I. Troponin I isoform switching thus occurs on the developing thin filament also.

Two-dimensional gel analysis of CL2-MF. Two-dimensional gel analysis of the CL2-MF isolated from 18-d-old embryonic rat hearts contained as major protein components $\alpha$-actin, the embryonic isoform of troponin $\mathrm{T}(E)$ and cardiac muscle $\mathrm{Tm}$ and 


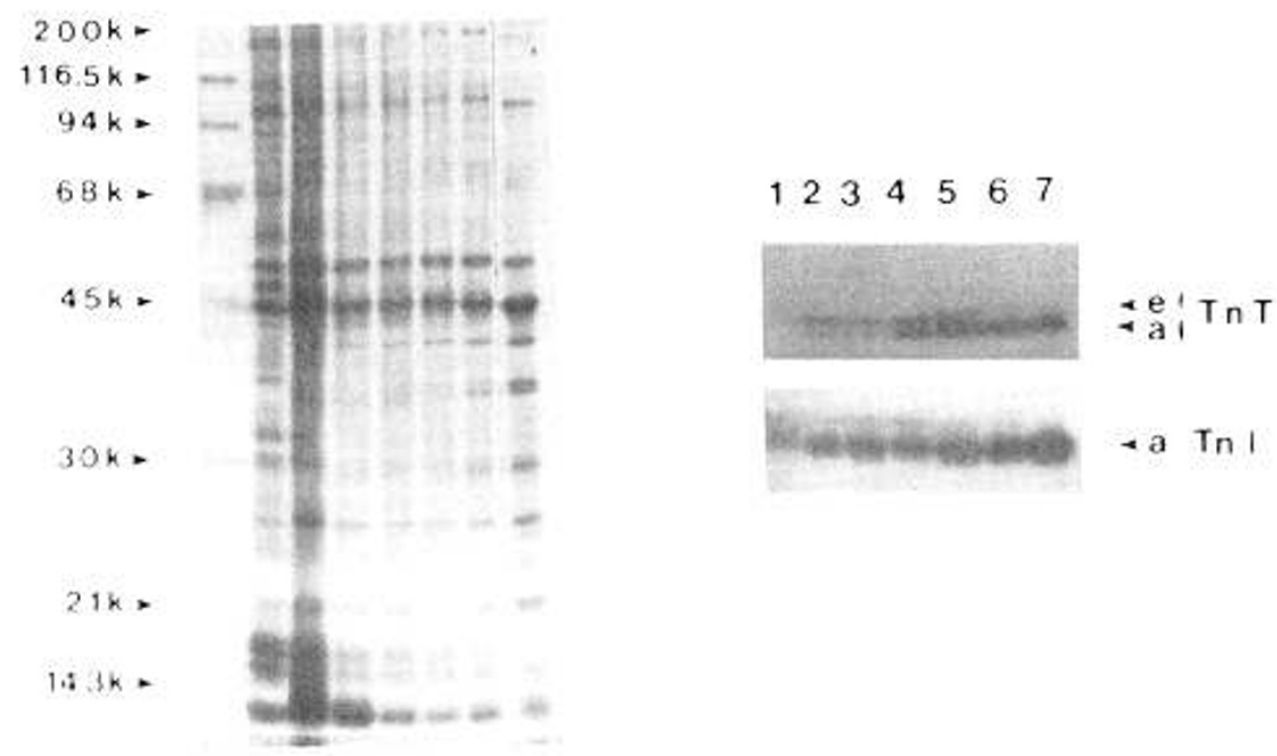

Fig. 4. Western immunoblot demonstrating troponin $\mathrm{T}$ isoform switching and the appearance of cardiac troponin I during rat heart development. After electrophoresis and transfer of proteins to nitrocellulose paper, the papers were either stained with amido black (left panel) or reacted with the polyclonal antibody RTI (bottom right panel) or with the MAb. CT3 (top right panel), followed by ${ }^{125}$ I-labeled goat anti-rabbit or goat anti-mouse $\mathrm{IgG}$, respectively. Bound antibody was detected by autoradiography. Heart homogenates are from d 16 embryos (lane 1), day of birth (lane 2), postnatal d 5 (lane 3), 10 (lane 4), 15 (lane 5), 20 (lane 6), and adult (lane 7). The embryonic isoform of troponin $\mathrm{T}(\mathrm{Tn} T$-e) is exclusively present in the late embryonic heart. On the day of birth, the adult isoform of troponin T ( $T n T-a)$ appears; as development progresses, this isoform increases, whereas the embryonic isoform decreases until it is undetectable after postnatal $\mathrm{d} 20$. Only the adult isoform of troponin I is consistently recognized $(T n I-a)$ by our polyclonal antibody; it is first detected in the embryo (lane 1 ) and increases in amount as development progresses.

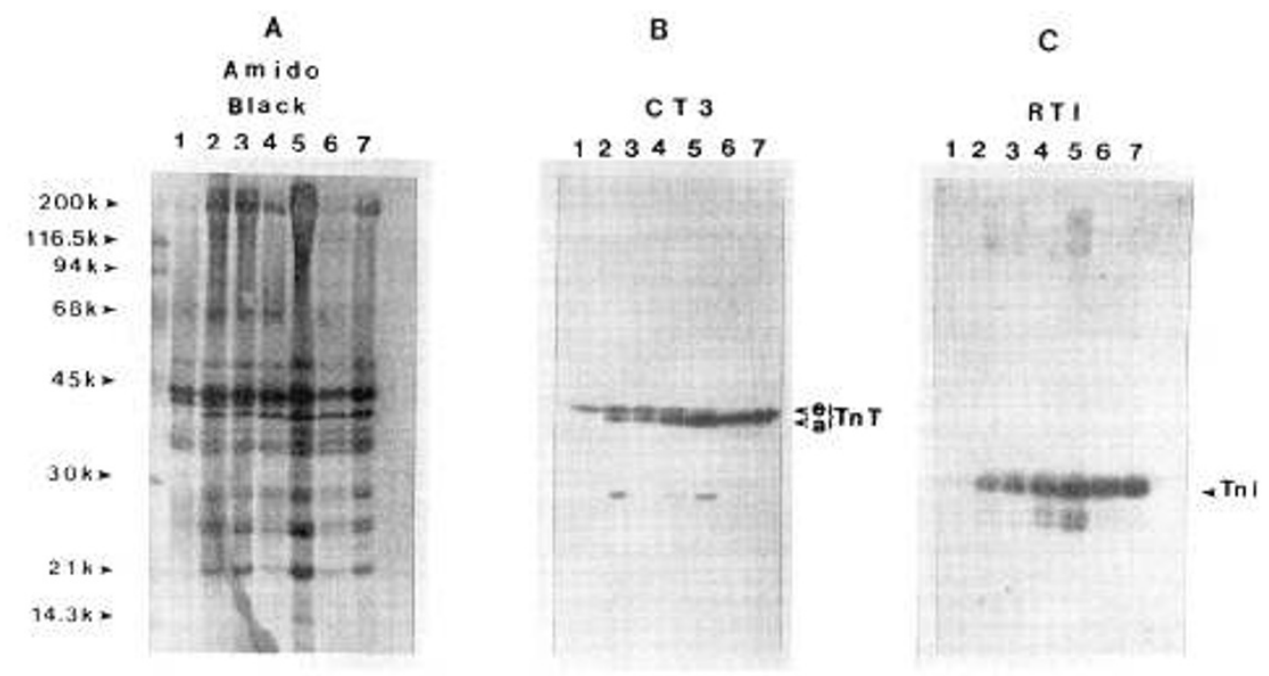

Fig. 5. Western immunoblot analysis of striated CL2-MF with antibodies to troponin T and troponin I. Western immunoblot was performed as described for Figure 4. Cardiac thin filaments were prepared (as described in Materials and Methods) from embryonic d 18 (lane 1), and postnatal ages $24 \mathrm{~h}$ (lane 2), $48 \mathrm{~h}$ (lane 3), $5 \mathrm{~d}$ (lane 4), $2 \mathrm{wk}$ (lane 5), $3 \mathrm{wk}$ (lane 6), and adult (lane 7). In panel B, troponin T isoform switching is demonstrated to occur on the thin filament starting immediately after birth and being completed by 3 wk of postnatal age, from the larger embryonic to the smaller adult isoform. The adult isoform of troponin I ( $T_{n} I$ in panel $\mathrm{C}$ ) is first detectable in thin filaments after birth, and it is found in increasing amount as development progresses.

pTM, as detected on a Coomassie blue-stained two-dimensional gel (Fig. $7 A$ ). At the stages of development examined, we are unable to detect any noncardiac isoform of actin; at least in chick hearts, it has been previously demonstrated in our lab (20) that skeletal muscle isoforms of actin are present on very early embryonic microfilaments, disappearing before hatching. Silver staining of the same gel reveals a minor protein spot in the region where the adult isoform of troponin $\mathrm{T}(\mathrm{Tn} T)$ would be expected to migrate (Fig. $7 B$ ). On the basis of Western immunoblots, it has been previously thought that the adult isoform of troponin
T appears only after birth (Fig. 4 and references 13 and 14). The appearance of the embryonic isoform of troponin $\mathrm{T}$ closer to the left side of the two-dimensional gel than the adult isoform indicates that it is a more acidic protein. Nonmuscle isoforms of $\operatorname{Tm} 3,4$, and 5 are also detected by silver staining of twodimensional gel analysis of thin filaments from embryonic rat hearts. As development progresses, the amount of the adult isoform of troponin $\mathrm{T}$ increases (Fig. $7 C$ and $E$ ), whereas the amount of embryonic troponin T decreases (Fig. $8 \mathrm{~A}$ ), disappearing before adulthood (Fig. $8 \mathrm{C}$ ). The apparent low abundance of 
A

Coomassie

Blue

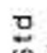

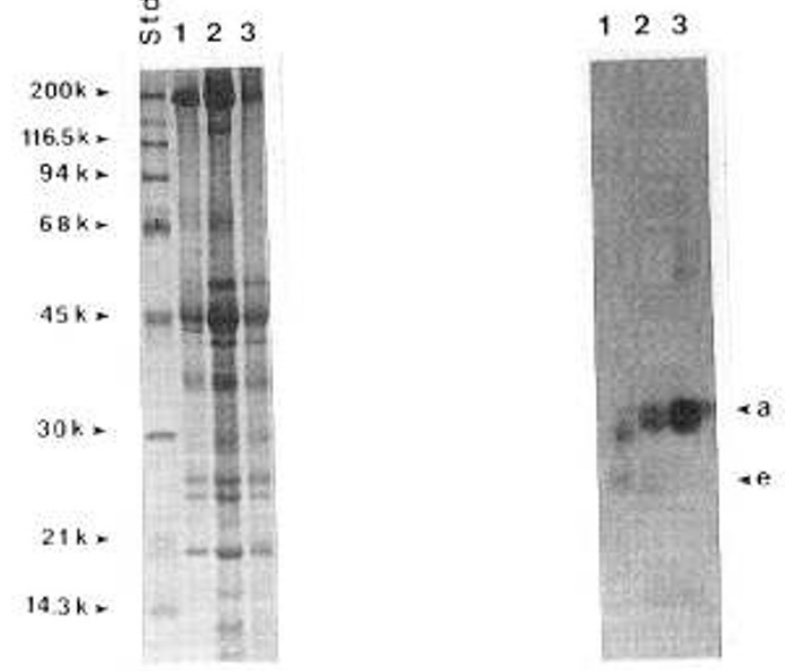

Fig. 6. Western immunoblot analysis of striated CL2-MF with an antibody to slow skeletal troponin I. (Western immunoblot performed as described for Fig. 4, except Coomassie blue stain of polyacrylamide gel is shown instead of amido black stain of nitrocellulose paper. ${ }^{125} \mathrm{I}$ labeled goat anti-guinea pig $\mathrm{IgG}$ was used as second antibody.) Cardiac thin filaments were prepared as described in Materials and Methods from embryonic d 18 (lane 1) and postnatal $\mathrm{d} 7$ (lane 2) and 21 (lane 3). Approximate loading amounts: lane $1,50 \mu \mathrm{g}$; lane 2, $100 \mu \mathrm{g}$; lane 3, $75 \mu \mathrm{g}$. (Loading amounts were chosen in an attempt to detect the embryonic isoform of troponin I at each age.) The adult isoform of troponin I $(a)$ is present on thin filaments from each age. The embryonic isoform of troponin I $(e)$ is detected on thin filaments from d 20 embryonic and postnatal 7 hearts but is not detectable on thin filaments by the 21 st postnatal d (lane 3 ).

adult troponin $\mathrm{T}$ seen on two-dimensional gels is likely related to the low solubility of this protein in low salt conditions present in our isoelectric focusing gel system. This phenomenon has been observed previously (33). In addition, troponin $\mathrm{T}$ aggregates are often seen in two-dimensional gel systems, reducing the apparent abundance of this protein. The presence of satellite protein spots around both isoforms of troponin $\mathrm{T}$ may represent charge heterogeneity, perhaps related to phosphorylation or other posttranslational modification. Charge heterogeneity of troponin $T$ is apparently present throughout development. Nonmuscle isoforms of Tm 4 and 5 persist in cardiac thin filaments throughout development (in silver-stained gels; see, e.g. Fig. $7 B, D$, and $F$ and Fig. $8 D$ ), whereas nonmuscle Tm isoform 3 disappears shortly after birth (Fig. $7 D$ and $F$ ). The degree of phosphorylation of $\mathrm{Tm}$ is greatest at the earliest stage of development examined (Fig. 7A); pTm represents the major species of Tm present at this stage of development. As development progresses, the degree of phosphorylation of $\mathrm{Tm}$ on thin filaments decreases, until the amount of Tm and $\mathrm{pTm}$ are apparently equal in adult microfilaments (compare, e.g. Fig. $7 A$ with Fig. $8 C$ ). This result is in agreement with the results of Montarras et al. (34) for developing chick skeletal muscle Tm, suggesting that the degree of phosphorylation detected is unlikely to be related to artifact during sample preparation. Several other minor spots on these twodimensional gels remain to be identified. Troponin I is not seen on these gels because it is such a basic protein.

\section{DISCUSSION}

Newborn myocardium is known to respond less dramatically than mature myocardium to inotropic agents. In a clinical situ-
A

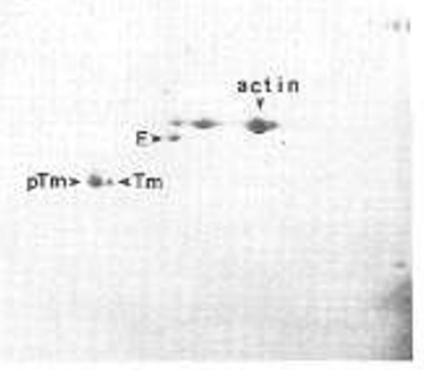

C
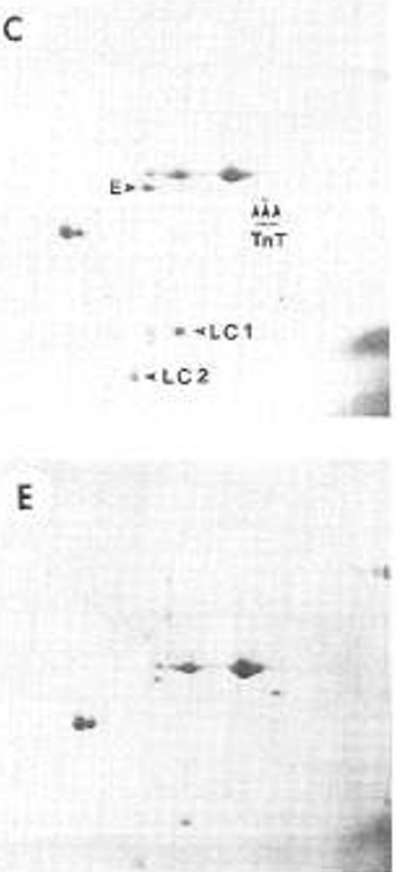

B

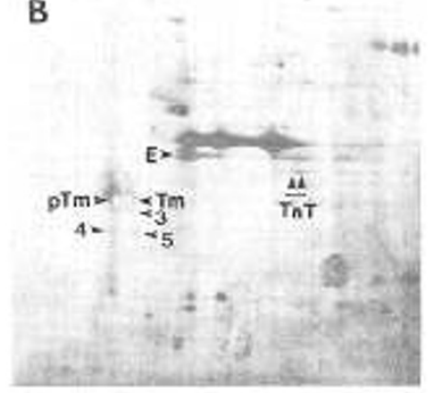

D

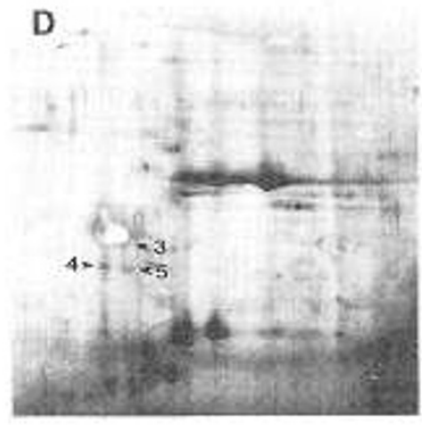

$F$

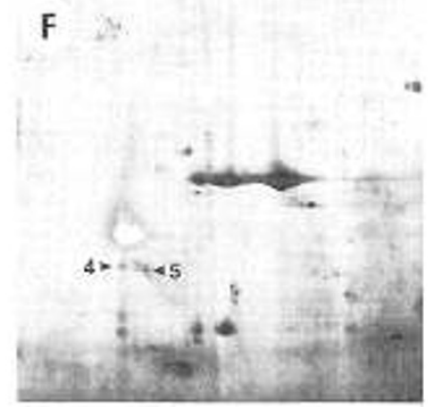

Fig. 7. Two-dimensional gel analysis of striated CL2-MF. Thin filaments prepared from hearts of $\mathrm{d} 18$ embryos $(A$ and $B)$, postnatal $\mathrm{d} 1$ ( $C$ and $D$ ), and postnatal 5 ( $E$ and $F$ ); panels $A, C$, and $E$ are Coomassie blue stained; panels $B, D$, and $F$ are silver stained. The 1 st dimension is isoelectric focusing with ampholine ( $\mathrm{pH} \mathrm{4-6)} \mathrm{(acidic} \mathrm{to} \mathrm{left,} \mathrm{basic} \mathrm{to}$ right); the second dimension is SDS-PAGE (larger proteins near the top). $E$, embryonic troponin T; 3,4 , and 5 , nonmuscle isoforms 3,4 , and 5 of $\mathrm{Tm}$; $T n T$, adult troponin $\mathrm{T}$. The degree of phosphorylation of $\mathrm{Tm}$ decreases as development progresses. The adult isoform of troponin $T$ is detected by silver stain in 18 embryonic cardiac thin filaments (TnT in panel B). Nonmuscle isoforms of Tm 4 and 5 are detected throughout development, whereas nonmuscle isoform 3 disappears after birth $(B, D$, and $F$ ).

ation, therefore, the augmentation of myocardial contractility can be more challenging in neonates than children or adults. It is likely that the profile of contractile proteins present in the contractile apparatus determines the functional capacity, i.e. ability to perform work. It is therefore attractive to attempt to explain developmental differences in myocardial function on the basis of sarcomeric protein isoform changes.

Troponin $\mathrm{T}$ isoform switching has been well described during rat heart development $(13,14)$. The larger embryonic isoform of troponin $\mathrm{T}$ is present exclusively during prenatal heart development. Shortly after birth (within the first $5 \mathrm{~d}$ ), the smaller adult isoform appears. As development progresses after birth, the adult isoform gradually replaces the embryonic isoform, the isoform switch being complete by 2 to $3 \mathrm{wk}$ postnatally. Furthermore, analysis of two-dimensional gels of immunoprecipitated thin filaments (Figs. 7 and 8) from late embryonic rat hearts demonstrates a small amount of the adult isoform of troponin $\mathrm{T}$ by 

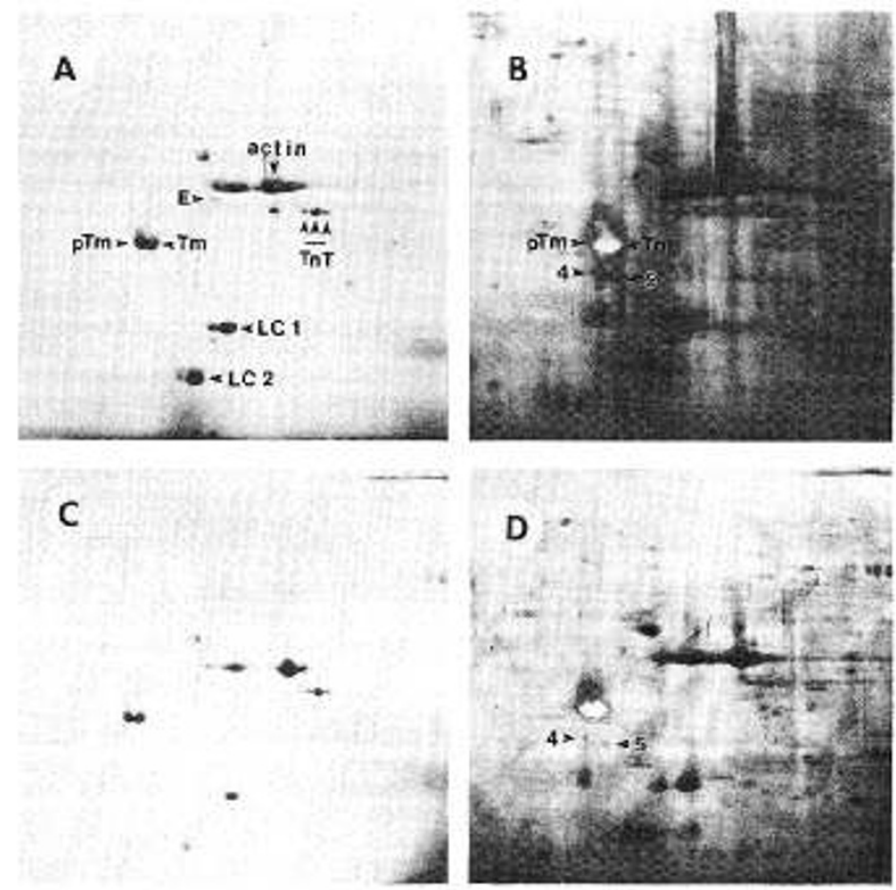

Fig. 8. Two-dimensional gel analysis of striated CL2-MF from 2-wk postnatal $(A$ and $B)$ and adult $(C$ and $D$ ) heart. (Two-dimensional gels run as described for Fig. 7). Abbreviations are as described for Figure 7 except $L C 1$ and $L C 2$ represent light chains 1 and 2, respectively, of myosin. The completion of troponin $\mathrm{T}$ isoform switching is demonstrated on the thin filament. Nonmuscle isoforms 4 and 5 of Tm are detected in thin filaments of both ages. The amount of non-pTm and pTm are approximately equal at these ages.

silver stain (Western immunoblots with the monoclonal CT3 antibody fail to demonstrate troponin $\mathrm{T}$ in the same-aged whole heart). These data suggest that troponin $\mathrm{T}$ isoform switching occurs on the developing thin filament but that the isoform switch actually begins before birth rather than in the neonatal period, as suggested in previous studies $(13,14)$. In chick heart, a single cardiac troponin $\mathrm{T}$ gene has been shown to produce two mRNA via developmentally regulated alternative splicing of a common primary transcript (35). The two mRNA differ in a single exon, and translation of these two mRNA generates the adult and embryonic isoforms of troponin $\mathrm{T}$. In rat cardiac muscle, mRNA data are not yet available. In preliminary work (currently in progress in our lab), Northern blot data appear to be in good agreement with protein data.

Tobacman and Lee (16) have found subtle differences in the calcium sensitivity of the actin-Tm-troponin complex between the two isoforms of troponin $\mathrm{T}$ purified from bovine hearts. If this difference is verified for species in which isoform switching of troponin $\mathrm{T}$ occurs (e.g. the rat) during development, then troponin $\mathrm{T}$ isoform switching may be responsible, at least in part, for developmental differences in myocardial function. Because no difference in its effect on Tm polymerization was noted between troponin $\mathrm{T}$ isoforms, it is difficult to ascribe a role for embryonic troponin $\mathrm{T}$ in assembling the developing thin filaments.

Troponin I isoform switching has also been investigated during rat heart development $(11,12)$. Before the 16 th $d$ of gestation, the smaller embryonic isoform of troponin I is exclusively present. Recent cloning data (32) demonstrate that the cDNA encoding this isoform has a high degree of sequence homology with the cDNA encoding troponin I from slow skeletal muscle, suggesting that the embryonic cardiac troponin I isoform is the same protein as slow skeletal troponin I. The adult troponin I isoform appears at about $16 \mathrm{~d}$ of gestation and gradually increases in amount as development progresses, until isoform switching is complete between the 2 nd and 3rd postnatal wk. Gene switching is thought to be the mechanism by which troponin I isoform switching occurs, rather than differential mRNA splicing, as for troponin $\mathrm{T}(32)$. The troponin I isoform switch has been postulated to modulate myocardial function via the presence on the adult isoform of an additional phosphorylation site (36). Phosphorylation at this site appears to alter the calcium affinity of troponin $\mathrm{C}$ by increasing the rate at which calcium can dissociate. In this fashion, troponin I phosphorylation may enhance sarcomeric relaxation $(17,18,36)$. Data presented in this study from developing rat heart thin filaments demonstrate that troponin I isoform switching appears to occur on the developing thin filament. Troponin I isoform switching is, therefore, also a phenomenon which may in part explain developmental differences in myocardial function. Because troponin $\mathrm{C}$ isoform switching has not been observed in mammalian heart, it is difficult to ascribe a significant role to this protein in determining developmental differences in myocardial function

The analysis of purified microfilaments rather than whole muscle is useful in that it demonstrates that troponin I and troponin $\mathrm{T}$ isoform switching occur on the thin filament. This excludes the possibility that embryonic troponin isoforms may be soluble proteins unassociated with the contractile apparatus. Whole tissue analysis assumes no soluble contractile proteins exist without providing direct evidence. In addition, analysis of purified microfilaments provides more sensitive data about proteins present in low abundance on the contractile apparatus, such as nonmuscle isoforms of Tm. In this fashion, information about assembly of the contractile apparatus during development can more readily be acquired because of the elimination (to a large extent) of noncontractile proteins during purification.

It has previously been described (37) that the degree of phosphorylation of $\mathrm{Tm}$ is highest in fetal heart tissue and decreases as development progresses. In the present study, two-dimensional gel analysis of CL2-MF demonstrates that this phenomenon occurs on the developing thin filament. This finding is consistent with earlier studies (20) performed in developing chick striated muscle.

The significance of phosphorylation of $\mathrm{Tm}$, and its change with development, is currently unknown. Heely et al. (38) have shown that the myosin ATPase activity in a reconstituted thin filament system in the presence of calcium is higher for $\mathrm{pTm}$ than non-pTm. This suggests that the degree of phosphorylation of Tm may represent a modulatory mechanism for the fine tuning of the contractile apparatus to the particular needs of myocardium at a given developmental age. In the same paper, it was reported that phosphorylation of Tm enhanced end-to-end Tm polymerization when compared with a system in which $\mathrm{Tm}$ was not phosphorylated. Tm is phosphorylated at a location (ser283 ) that has been shown to be involved in key functions of $\mathrm{Tm}$ including the interaction with troponin $\mathrm{T}$ (39) and cooperative binding of Tm to actin filaments (40). Phosphorylation of Tm may thus play a role in facilitation of thin filament formation during myofibrillogenesis.

The coexistence of cardiac and nonmuscle isoforms of $\mathrm{Tm}$ in thin filaments from developing and mature hearts is in good agreement with data from our laboratory (20) examining in ovo chick muscle development. At least one of the nonmuscle $\mathrm{Tm}$ isoforms (isoform 3) appeared to decrease and disappear during the maturation process. We speculate that nonmuscle isoforms of Tm may play an assembly role during thin filament differentiation. The possibility that nonmuscle isoforms of Tm may represent a set of functionally distinct microfilaments in differentiating cardiac muscle cells cannot, however, be excluded by the present approach.

\section{REFERENCES}

1. Park I-S, Michael LH, Driscoll DJ 1982 Comparative response of the developing canine myocardium to inotropic agents. Am J Physiol 242:H13-H18 2. Park MK, Sheridan PH, Morgan WF, Bick N 1980 Comparative inotropic 
response of newborn and adult rabbit papillary muscles to isoproteronol and calcium. Dev Pharmacol Ther 1:70-82

3. Hopkins Jr SF, McCutcheon EP, Wekstein DR 1973 Postnatal changes in rat ventricular function. Circ Res 32:685-691

4. Binah O, Legato MJ, Danillo P, Rosen MR 1983 Developmental changes in the cardiac effects of amrinone in the dog. Circ Res 52:747-752

5. Murphy AM, Solaro RJ 1990 Developmental difference in the stimulation of cardiac myofibrillar Mg ATPase activity by calmidazolium. Pediatr Res 28:46-49

6. Solaro RJ, Lee JA, Kentish JC, Allen DG 1988 Effects of acidosis on ventricular muscle from adult and neonatal rats. Circ Res 63:774-787

7. Caplan AI, Fiszman MY, Eppenberger HM 1983 Molecular and cell isoforms during development. Science 221:921-927

8. Swynghedauw B 1986 Developmental and functional adaptation of contractile proteins in cardiac and skeletal muscles. Physiol Rev 66:710-771

9. Hoh JFY, McGrath PA, Hale PT 1977 Electrophoretic analysis of multiple forms of rat cardiac myosin: effects of hypophysectomy and thyroxine replacement. J Mol Cell Cardiol 10:1053-1076

10. Mahdavi V, Lompre AM, Chambers AP, Nadal-Ginard B 1984 Cardiac myosin heavy chain isozymic transitions during development and under pathological conditions are regulated at the level of mRNA availability. Eur Heart $\mathrm{J}$ 5(suppl F): 181-191

11. Saggin L, Gorza L, Ausoni S, Schiaffino S 1989 Troponin I switching in the developing heart. J Biol Chem 264:16299-16302

12. Sabry MA, Dhoot GK 1989 Identification and pattern of expression of a developmental isoform of troponin I in chicken and rat cardiac muscle. $J$ developmental isoform of troponin
Muscle Res Cell Motil 10:85-91

13. Jin JP, Lin JJ-C 1988 Rapid purification of mammalian cardiac troponin $T$ and its isoform switching during development. J Biol Chem 263:7309-7315

14. Saggin L, Ausoni S, Gorza L, Sartore S, Schiaffino S 1988 Troponin T switching in the developing rat heart. J Biol Chem 263:18488-18492

15. Morkin E, Bahl JJ, Flink IL, Markham BE, Tsika RW 1990 Molecular analysis of human and rat alpha myosin heavy chain genes. In: Chien S (ed) Molecular Biology of the Cardiovascular System. Lea and Febiger, Philadelphia, pp 4551

16. Tobacman LS, Lee R 1987 Isolation and functional comparison of bovine troponin $\mathrm{T}$ isoforms. J Biol Chem 262:4059-4064

17. Wilkinson JM, Grand RJA 1978 Comparison of amino acid sequence of troponin I from different striated muscles. Nature 271:31-35

18. Solaro RJ, Moir AJG, Perry SV 1976 Phosphorylation of troponin I and the inotropic effect of adrenaline in the perfused rabbit heart. Nature 262:615616

19. Lin JJ-C, Lin JL-C 1986 Assembly of different isoforms of actin and tropomyosin into the skeletal tropomyosin-enriched microfilaments during differentiation of muscle cells in vitro. J Cell Biol 103:2173-2183

20. Wang S-M, Wang S-H, Lin JL-C, Lin JJ-C 1990 Striated muscle tropomyosinenriched microfilaments of developing muscles of chicken embryos. J Muscle Res Cell Motil 11:191-202

21. Potter JD 1982 Preparation of troponin and its subunits. Methods Enzymol $85: 241-259$
22. Harlow E, Lane D 1988 Electroelution of protein antigens from polyacrylamide gel slices. In: Harlow E, Lane D (eds) Antibodies, A Laboratory Manual. Cold Spring Harbor Laboratory, Cold Spring Harbor, NY, p 70

23. Towbin H, Staehelin T, Gordon J 1979 Electrophoretic transfer of proteins from polyacrylamide gels to nitrocellulose sheets: procedure and some applications. Proc Natl Acad Sci USA 76:4350-4354

24. Lazarides E, Weber K 1974 Actin antibody: the specific visualization of actin filaments in non-muscle cells. Proc Natl Acad Sci USA 71:2268-2272

25. Jin JP, Lin JL-C, Lin JJ-C 1990 Troponin T isoform switching during heart development. Ann NY Acad Sci 588:393-396

26. Lin JJ-C, Chou C-S, Lin JL-C 1985 Monoclonal antibodies against chicken tropomyosin isoforms: production, characterization, and application. Hybridoma 4:223-242

27. Borg TK, Rubin K, Lundgren E, Borg K, O'Brink B 1984 Recognition of extracellular matrix components by neonatal and adult cardiac myocytes. Dev Biol 104:86-96

28. Laemmli UK 1970 Cleavage of structural proteins during the assembly of the head of bacteriophage T4. Nature 227:680-685

29. Blattler DP, Garner F, van Slyke K, Bradley A 1972 Quantitative electrophoresis in polyacrylamide gels of $2-40 \%$. J Chromatogr $64: 147-155$

30. Lin JJ-C, Matsamura F, Yamashiro-Matsamura S 1984 Tropomyosin-enriched and alpha actinin-enriched microfilaments isolated from chicken embryo fibroblasts by monoclonal antibodies. J Cell Biol 98:116-127

31. O'Farrell PH 1975 High resolution two-dimensional electrophoresis of proteins. J Biol Chem 250:4007-402

32. Murphy AM, Dieckman LJ, Solaro RJ, Strauss AW 1990 Gene switching regulates troponin I expression in rat cardiac development; influence of thyroid status. Pediatr Res 27:236A(abstr)

33. Greaser ML, Gergley J 1972 Purification and properties of the components from troponin. J Biol Chem 248:2125-2183

34. Montarras D, Fiszman MY, Gros F 1982 Changes in tropomyosin during development of chick embryonic skeletal muscles in vivo and during differentiation of chick muscle cells in vitro. J Biol Chem 257:545-548

35. Cooper TA, Ordahl CP 1985 A single cardiac troponin T gene generates embryonic and adult isoforms via developmentally regulated alternate splicing. J Biol Chem 260:11140-11148

36. Robertson SP, Johnson JD, Holroyde MJ, Kramer EG, Potter JD, Solaro RJ 1982 The effect of troponin I phosphorylation on the calcium binding properties of the calcium regulatory site of bovine cardiac troponin. J Biol Chem 257:260-263

37. Heely DA, Moir AJG, Perry SV 1982 Phosphorylation of tropomyosin during development in mammalian striated muscle. FEBS Lett 146:115-118

38. Heely DA, Watson MH, Mak AS, Dubord P, Smillie LB 1989 Effect of phosphorylation on the interaction and functional properties of rabbit striated muscle alpha tropomyosin. J Biol Chem 264:2424-2430

39. Brisson JR, Golosinka K, Smillie LB, Sykes BD 1986 Interaction of tropomyosin and troponin T: a protein nuclear magnetic resonance study. Biochemistry 25:4548-4555

40. Mak AS, Smillie LB 1981 Structural interpretation of the two site binding of troponin on the muscle thin filament. J Mol Biol 149:541-550 